\title{
Y-TYPE FLUX-TUBE FORMATION AND GLUONIC EXCITATIONS IN BARYONS: FROM QCD TO QUARK MODEL
}

\author{
HIDEO SUGANUMA AND HIROKO ICHIE \\ Faculty of Science, Tokyo Institute of Technology, \\ Ohokayama 2-12-1, Meguro, Tokyo 152-8551, Japan \\ suganuma@th.phys.titech.ac.jp \\ TORU T. TAKAHASHI \\ Yukawa Institute for Theoretical Physics, Kyoto University, \\ Kitashirakawa-Oiwake, Sakyo, Kyoto 606-8502, Japan
}

\begin{abstract}
Using SU(3) lattice QCD, we perform the first systematic study for the groundstate three-quark (3Q) potential $V_{3 \mathrm{Q}}^{\text {g.s. }}$ and the 1 st excited-state $3 \mathrm{Q}$ potential $V_{3 \mathrm{Q}}^{\text {e.s. }}$, i.e., the energies of the ground state and the 1st excited state of the gluon field in the presence of the static three quarks. From the accurate and thorough calculation for more than 300 different patterns of 3Q systems, the static ground-state $3 \mathrm{Q}$ potential $V_{3 \mathrm{Q}}^{\text {g.s. }}$ is found to be well described by the Coulomb plus Y-type linear potential, i.e., Y-Ansatz, within 1\%-level deviation. As a clear evidence for Y-Ansatz, Y-type flux-tube formation is actually observed on the lattice in maximally-Abelian projected QCD. For more than 100 patterns of 3Q systems, we calculate the 1 st excited-state $3 \mathrm{Q}$ potential $V_{3 \mathrm{Q}}^{\text {e.s. }}$ in quenched lattice $\mathrm{QCD}$, and find the gluonic excitation energy $\Delta E_{3 \mathrm{Q}} \equiv V_{3 \mathrm{Q}}^{\text {e.s. }}-V_{3 \mathrm{Q}}^{\text {g.s. }}$ to be about $1 \mathrm{GeV}$. This large gluonic-excitation energy is conjectured to ensure the success of the quark model for the low-lying hadrons even without gluonic excitations.
\end{abstract}

\section{Introduction $\sim$ Hadron Physics based on QCD}

Quantum chromodynamics (QCD), the SU(3) gauge theory, was first proposed by Yoichiro $\mathrm{Nambu}^{1}$ in 1966 as a candidate for the fundamental theory of the strong interaction, just after the introduction of the "new" quantum number, "color". ${ }^{2}$ In spite of its simple form, QCD creates thousands of hadrons and makes the vacuum nontrivial, which leads to various interesting nonperturbative phenomena such as color confinement ${ }^{3}$ and dynamical chiral-symmetry breaking. ${ }^{4}$ This miracle of QCD is due to its strong-coupling nature in the infrared region, but the strong-coupling nature itself makes very difficult to deal with QCD. 
In recent years, according to the remarkable progress of the computational power, the lattice QCD Monte Carlo calculation becomes a reliable and useful method for the analysis of nonperturbative QCD, ${ }^{5}$ which indicates an important direction in the hadron physics. In this paper, using lattice QCD, we study the three-quark potential in detail. $6,7,8,9$

\section{The Ground-State Three-Quark Potential in QCD}

In general, the three-body force is regarded as a residual interaction in most fields in physics. In QCD, however, the three-body force among three quarks is a "primary" force reflecting the $\mathrm{SU}(3)$ gauge symmetry. In fact, the three-quark $(3 \mathrm{Q})$ potential is directly responsible for the structure and properties of baryons, similar to the relevant role of the $\mathrm{Q}-\overline{\mathrm{Q}}$ potential for meson properties, and both the $\mathrm{Q}-\overline{\mathrm{Q}}$ potential and the $3 \mathrm{Q}$ potential are equally important fundamental quantities in QCD. Furthermore, the $3 \mathrm{Q}$ potential is the key quantity to clarify the quark confinement in baryons. However, in contrast with a number of studies on the $\mathrm{Q}-\overline{\mathrm{Q}}$ potential using lattice $\mathrm{QCD},{ }^{5,10}$ there was almost no lattice QCD study for the $3 \mathrm{Q}$ potential before our study in $1999,{ }^{9}$ in spite of its importance in the hadron physics.

\subsection{Theoretical Form for the $3 Q$ Potential $\sim$ Y-Ansatz}

From the detailed studies with lattice QCD, the Q-Q potential is known to be well described with the inter-quark distance $r$ as ${ }^{5,7}$

$$
V_{\mathrm{Q} \overline{\mathrm{Q}}}(r)=-\frac{A_{\mathrm{Q} \overline{\mathrm{Q}}}}{r}+\sigma_{\mathrm{Q} \overline{\mathrm{Q}}} r+C_{\mathrm{Q} \overline{\mathrm{Q}}} .
$$

As for the $3 \mathrm{Q}$ potential form, there are two theoretical arguments for the limits of short and long distances.

(1) At the short distance, perturbative QCD is applicable, and therefore $3 \mathrm{Q}$ potential is expressed as the sum of the two-body Coulomb potential originating from the one-gluon-exchange process.

(2) At the long distance, the strong-coupling expansion of QCD is plausible, and it leads to the flux-tube picture. ${ }^{11}$ For the $3 \mathrm{Q}$ system, there appears a junction which connects the three flux-tubes from the three quarks, and Y-type flux-tube formation is expected. ${ }^{12,13,14}$

Then, we theoretically conjecture the functional form of the $3 \mathrm{Q}$ potential as the Coulomb plus Y-type linear potential, i.e., Y-Ansatz,

$$
V_{3 \mathrm{Q}}^{\mathrm{g} . \mathrm{s}}=-A_{3 \mathrm{Q}} \sum_{i<j} \frac{1}{\left|r_{i}-r_{j}\right|}+\sigma_{3 \mathrm{Q}} L_{\min }+C_{3 \mathrm{Q}}
$$


where $L_{\min }$ is the minimal value of the total flux-tube length given by

$$
\begin{aligned}
& L_{\min }=\overline{\mathrm{AP}}+\overline{\mathrm{BP}}+\overline{\mathrm{CP}} \\
= & \left(\frac{1}{2}\left(a^{2}+b^{2}+c^{2}\right)+\frac{\sqrt{3}}{2} \sqrt{(-a+b+c)(a-b+c)(a+b-c)(a+b+c)}\right)^{1 / 2}
\end{aligned}
$$

as shown in Fig.1.

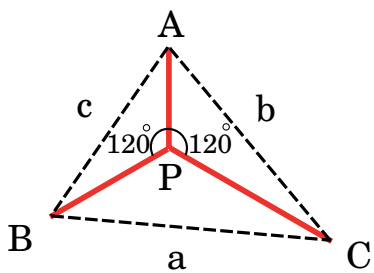

Figure 1. The flux-tube configuration of the $3 \mathrm{Q}$ system with the minimal value $L_{\min }$ of the total flux-tube length. There appears a physical junction linking the three flux-tubes at the Fermat point $\mathrm{P}$, and one finds $L_{\min }=\overline{\mathrm{AP}}+\overline{\mathrm{BP}}+\overline{\mathrm{CP}}$.

Of course, it is nontrivial that these simple arguments on UV and IR limits of QCD hold for the intermediate region as $0.2 \mathrm{fm}<r<1 \mathrm{fm}$. Then, we study the $3 \mathrm{Q}$ potential in lattice QCD. Note that the lattice QCD data itself is completely independent of any Ansatz for the potential form.

\subsection{The Three-Quark Wilson Loop}

Similar to the Q- $\bar{Q}$ potential calculated with the Wilson loop, the $3 \mathrm{Q}$ potential can be calculated with the $3 \mathrm{Q}$ Wilson loop ${ }^{6,7,8,13,14}$ defined on the contour of three large staples as

$$
W_{3 \mathrm{Q}} \equiv \frac{1}{3 !} \epsilon_{a b c} \epsilon_{a^{\prime} b^{\prime} c^{\prime}} U_{1}^{a a^{\prime}} U_{2}^{b b^{\prime}} U_{3}^{c c^{\prime}}
$$

with $U_{k} \equiv P \exp \left\{i g \int_{\Gamma_{k}} d x_{\mu} A^{\mu}(x)\right\}$ in Fig.2. The 3Q Wilson loop physically means that a color-singlet gauge-invariant $3 \mathrm{Q}$ state is created at $t=0$ and is annihilated at $t=T$ with the three quarks spatially fixed for $0<t<T$.

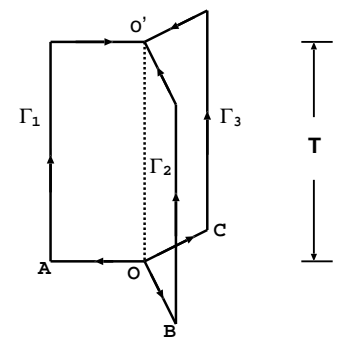

Figure 2. The 3Q Wilson loop. A color-singlet (gauge-invariant) 3Q state is created at $t=0$ and is annihilated at $t=T$. The three quarks are spatially fixed for $0<t<T$. 
The vacuum expectation value of the $3 \mathrm{Q}$ Wilson loop is expressed as

$$
\left\langle W_{3 \mathrm{Q}}\right\rangle=\sum_{n=0}^{\infty} C_{n} \exp \left(-V_{n} T\right),
$$

where $V_{n}$ denotes the $n$-th energy of the gauge-field configuration in the presence of the spatially-fixed three quarks. ${ }^{6,7,8}$

It is worth mentioning that, while $V_{n}$ depends only on the $3 \mathrm{Q}$ location, $C_{n}$ depends on the operator choice at $t=0$ and $T, e . g$. , path linking between $3 \mathrm{Q}$. In fact, the gauge-invariant $3 \mathrm{Q}$ state prepared at $t=0$ generally includes excited-state contributions. In principle, by taking the large $T$ limit, the ground-state potential $V_{0}$ can be extracted as $\left\langle W_{3 \mathrm{Q}}\right\rangle \sim C_{0} \exp \left(-V_{0} T\right)$. However, the large- $T$ limit calculation is difficult in the practical latticeQCD calculation, since the signal decreases exponentially with $T$. Therefore, for the accurate calculation, it is desired to reduce the excited-state component in the 3Q state prepared at $t=0$ and $T$.

\subsection{Smearing Method: Excited-State Component Reduction}

The smearing method is one of the most popular and useful techniques to extract the ground state in lattice QCD, ${ }^{7}$ and is actually successful for the ground-state $\mathrm{Q}-\overline{\mathrm{Q}}$ potential. ${ }^{7}$ The standard smearing for link-variables is expressed as the iterative replacement of the spatial link-variable $U_{i}(s)$ $(i=1,2,3)$ by the obscured link-variable $\bar{U}_{i}(s) \in \mathrm{SU}(3)$ which maximizes

$$
\operatorname{Re} \operatorname{tr}\left[\bar{U}_{i}^{\dagger}(s)\left\{\alpha U_{i}(s)+\sum_{j \neq i, \pm} U_{ \pm j}(s) U_{i}(s \pm \hat{j}) U_{ \pm j}^{\dagger}(s+\hat{i})\right\}\right]
$$

with the smearing parameter $\alpha \in \mathbf{R}$. Here, we define $U_{-\mu}(s) \equiv U_{\mu}^{\dagger}(s-\hat{\mu})$. This procedure is schematically illustrated in Fig.3. The $n$-th smeared linkvariables $U_{\mu}^{(n)}(s)$ are iteratively defined as $U_{i}^{(n)}(s) \equiv \bar{U}_{i}^{(n-1)}(s)(i=1,2,3)$ and $U_{4}^{(n)}(s) \equiv U_{4}(s)$ starting from $U_{\mu}^{(0)}(s) \equiv U_{\mu}(s)$.

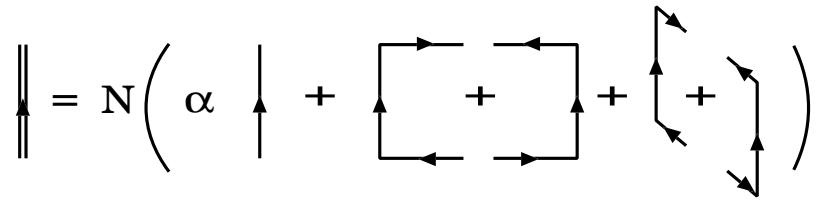

Figure 3. The schematic illustration for the smearing method for the link-variables.

Note that the smearing is just a method to construct the operator, and hence it never changes the physics itself such as the gauge configuration, unlike the cooling. As an important feature, the gauge-transformation property of $U_{\mu}^{(n)}(s)$ is just the same as that of $U_{\mu}(s)$, which ensures the gauge invariance of the $n$-th smeared $3 \mathrm{Q}$ Wilson loop $\left\langle W_{3 \mathrm{Q}}\left[U_{\mu}^{(n)}(s)\right]\right\rangle$. 
The fat link-variable $U_{\mu}^{(n)}(s)$ includes a spatial extension in terms of the original link-variable $U_{\mu}(s)$, and the smeared "line" expressed with $U_{\mu}^{(n)}(s)$ physically corresponds to a Gaussian-distributed "flux-tube". ${ }^{7}$ Therefore, the properly smeared line is expected to resemble the ground-state fluxtube. Here, the smearing parameter $\alpha$ and the iteration number $n$ can be regarded as the variational parameters to enhance the ground-state overlap.

Thus, through the selection of the properly smeared 3Q Wilson loop $\left\langle W_{3 \mathrm{Q}}\left[U_{\mu}^{(n)}(s)\right]\right\rangle$, we can construct the ground-state-dominant 3Q operator for the accurate measurement of the ground-state $3 \mathrm{Q}$ potential. ${ }^{6,7,8}$

\subsection{Lattice $Q C D$ results for the Ground-State $3 Q$ Potential}

For more than 300 different patterns of spatially-fixed 3Q systems, we perform the thorough calculation of the ground-state potential $V_{3 \mathrm{Q}}^{\text {g.s. }}$ in $\mathrm{SU}(3)$ lattice QCD with the standard plaquette action with $12^{3} \times 24$ at $\beta=5.7$ and with $16^{3} \times 32$ at $\beta=5.8$ and 6.0 at the quenched level. For the accurate measurement, we use the smearing method and construct the ground-statedominant $3 \mathrm{Q}$ operator. ${ }^{6,7,8}$

To conclude, we find that the static ground-state $3 \mathrm{Q}$ potential $V_{3 \mathrm{Q}}^{\text {g.s. }}$ is well described by the Coulomb plus Y-type linear potential, i.e., Y-Ansatz, within 1\%-level deviation, ${ }^{6,7}$ as shown in Table 1.

Table 1. Examples of the $3 \mathrm{Q}$ potential $V_{3 \mathrm{Q}}^{\text {latt }}$ for the $3 \mathrm{Q}$ system put on $(i, 0,0),(0, j, 0),(0,0, k)$ in $\mathbf{R}^{3}$ in lattice $\mathrm{QCD}$ at $\beta=6.0$. For each $3 \mathrm{Q}$ configuration, $V_{3 \mathrm{Q}}^{\text {latt }}$ is measured from the singleexponential fit as $\left\langle W_{3 \mathrm{Q}}\right\rangle=\bar{C} e^{-V_{3 \mathrm{Q}} T} . \bar{C} \simeq 1$ physically means the ground-state dominance in the smeared 3Q Wilson loop. We add the difference from the best-fit Y-Ansatz, $\Delta V \equiv\left|V_{3 \mathrm{Q}}^{\text {latt }}-V_{3 \mathrm{Q}}^{\mathrm{Y}}\right|$, which is only about $1 \%$ of the typical scale of $V_{3 Q}$. The listed value is measured in the lattice unit.

\begin{tabular}{cccccccc}
\hline \hline$(i, j, k)$ & $V_{3 \mathrm{Q}}^{\text {latt }}$ & $\bar{C}$ & $\Delta V$ & $(i, j, k)$ & $V_{3 \mathrm{Q}}^{\text {latt }}$ & $\bar{C}$ & $\Delta V$ \\
\hline$(0,1,1)$ & $0.6778(6)$ & $0.9784(24)$ & 0.0012 & $(0,2,3)$ & $1.0259(24)$ & $0.9607(91)$ & 0.0045 \\
$(0,1,2)$ & $0.8234(11)$ & $0.9712(45)$ & 0.0042 & $(0,2,4)$ & $1.0946(32)$ & $0.9657(120)$ & 0.0003 \\
$(0,1,3)$ & $0.9183(17)$ & $0.9769(65)$ & 0.0045 & $(0,2,5)$ & $1.1454(41)$ & $0.9282(149)$ & 0.0064 \\
$(0,1,4)$ & $0.9859(24)$ & $0.9589(92)$ & 0.0050 & $(0,2,6)$ & $1.2075(28)$ & $0.9464(76)$ & 0.0018 \\
$(0,1,5)$ & $1.0463(30)$ & $0.9495(112)$ & 0.0064 & $(0,2,7)$ & $1.2563(33)$ & $0.9262(90)$ & 0.0012 \\
$(0,1,6)$ & $1.1069(40)$ & $0.9595(152)$ & 0.0122 & $(0,3,3)$ & $1.0999(23)$ & $0.9566(62)$ & 0.0031 \\
$(0,1,7)$ & $1.1572(50)$ & $0.9374(192)$ & 0.0102 & $(0,3,4)$ & $1.1595(25)$ & $0.9454(67)$ & 0.0044 \\
$(0,2,2)$ & $0.9430(21)$ & $0.9586(78)$ & 0.0095 & $(0,3,5)$ & $1.2170(25)$ & $0.9426(65)$ & 0.0026 \\
\hline \hline
\end{tabular}

We summarize in Table 2 the lattice QCD results for the string tension and the Coulomb coefficient, with comparing between $3 \mathrm{Q}$ and $\mathrm{Q}-\overline{\mathrm{Q}}$ potentials. As remarkable features, we find the universality of the string tension between the $3 \mathrm{Q}$ and $\mathrm{Q}-\overline{\mathrm{Q}}$ systems and the one-gluon-exchange result as ${ }^{6,7}$

$$
\sigma_{3 \mathrm{Q}} \simeq \sigma_{\mathrm{Q} \overline{\mathrm{Q}}}, \quad A_{3 \mathrm{Q}} \simeq \frac{1}{2} A_{\mathrm{Q} \overline{\mathrm{Q}}} .
$$


Table 2. The best-fit parameter set $(\sigma, A, C)$ in the function form of Y-Ansatz, $V_{3 \mathrm{Q}}=-A_{3 \mathrm{Q}} \sum_{i<j} \frac{1}{\left|\mathbf{r}_{i}-\mathbf{r}_{j}\right|}+\sigma_{3 \mathrm{Q}} L_{\mathrm{min}}+C_{3 \mathrm{Q}}$, where $L_{\mathrm{min}}$ denotes the minimal value of the Y-type flux-tube length. The similar fit on the $\mathrm{Q}-\overline{\mathrm{Q}}$ potential (on-axis) is also listed. The physical unit is determined so as to reproduce $\sqrt{\sigma}=427 \mathrm{MeV}$ for the $\mathrm{Q}-\overline{\mathrm{Q}}$ potential. The universality of the string tension and the OGE result on the Coulomb coefficient are found as $\sigma_{3 \mathrm{Q}} \simeq \sigma_{\mathrm{QQ}}$ and $A_{3 \mathrm{Q}} \simeq \frac{1}{2} A_{\mathrm{Q} \overline{\mathrm{Q}}}$, respectively.

\begin{tabular}{lllcll}
\hline \hline & & $\sigma\left[a^{-2}\right]$ & $\sqrt{\sigma}[\mathrm{MeV}]$ & \multicolumn{1}{c}{$A$} & \multicolumn{1}{c}{$C\left[a^{-1}\right]$} \\
\hline$\beta=5.7$ & $3 \mathrm{Q}_{\mathrm{Y}}$ & $0.1524(28)$ & 413.0 & $0.1331(66)$ & $0.9182(213)$ \\
$(a \simeq 0.186 \mathrm{fm})$ & $\mathrm{Q} \overline{\mathrm{Q}}$ & $0.1629(47)$ & 427 & $0.2793(116)$ & $0.6203(161)$ \\
\hline$\beta=5.8$ & $3 \mathrm{Q}_{\mathrm{Y}}$ & $0.1027(6)$ & 416.6 & $0.1230(20)$ & $0.9085(55)$ \\
$(a \simeq 0.152 \mathrm{fm})$ & $\mathrm{Q} \overline{\mathrm{Q}}$ & $0.1079(28)$ & 427 & $0.2607(174)$ & $0.6115(197)$ \\
\hline$\beta=6.0$ & $3 \mathrm{Q}_{\mathrm{Y}}$ & $0.0460(4)$ & 407.1 & $0.1366(11)$ & $0.9599(35)$ \\
$(a \simeq 0.104 \mathrm{fm})$ & $\mathrm{Q} \overline{\mathrm{Q}}$ & $0.0506(7)$ & 427 & $0.2768(24)$ & $0.6374(30)$ \\
\hline \hline
\end{tabular}

\subsection{Further Investigations with various Ansätze}

Lattice Coulomb plus Y-type Linear Ansatz: Considering the lattice discretization error at a very short distance, we perform also more accurate fitting with the lattice Coulomb plus Y-type linear potential. We obtain almost the same result and confirm that Y-Ansatz is correct. ${ }^{7}$

Generalized Y-type Linear Ansatz : Considering a possible contamination of the $\Delta$-type flux at a short distance, we study generalized Y-Ansatz, ${ }^{7}$ which includes both $\mathrm{Y}$ and $\Delta$ Ansätze in some limits. The flux-tube core radius $R$ is found to be rather small as $R \simeq 0.08 \mathrm{fm}$, which again supports Y-Ansatz. ${ }^{7}$ (See Fig.4.)

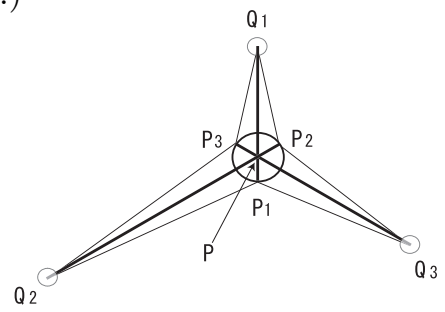

Figure 4. Generalized Y-Ansatz. The three quarks are spatially fixed at $\mathrm{Q}_{1}, \mathrm{Q}_{2}, \mathrm{Q}_{3}$. The point $\mathrm{P}_{1}$ is taken inside the circle around the physical junction (Fermat point) $\mathrm{P}$ so as to minimize $\mathrm{P}_{1} \mathrm{Q}_{2}+\mathrm{P}_{1} \mathrm{Q}_{3} . \mathrm{P}_{2}$ and $\mathrm{P}_{3}$ are similarly defined. In generalized Y-Ansatz, $\overline{L_{\text {min }}} \equiv \frac{1}{2}\left(\overline{\mathrm{P}_{1} \mathrm{Q}_{2}}+\overline{\mathrm{P}_{1} \mathrm{Q}_{3}}+\overline{\mathrm{P}_{2} \mathrm{Q}_{1}}+\overline{\mathrm{P}_{2} \mathrm{Q}_{3}}+\overline{\mathrm{P}_{3} \mathrm{Q}_{1}}+\overline{\mathrm{P}_{3} \mathrm{Q}_{2}}\right)$ is used instead of $L_{\text {min }}$

Yukawa plus Y-type Linear Ansatz: As other type of generalization, we investigate also the Yukawa plus Y-type linear potential,

$$
V_{3 \mathrm{Q}}=-A_{3 \mathrm{Q}}^{\text {Yukawa }} \sum_{i<j} \frac{e^{-m\left|r_{i}-r_{j}\right|}}{\left|r_{i}-r_{j}\right|}+\sigma_{3 \mathrm{Q}}^{\text {Yukawa }} L_{\min }+C_{3 \mathrm{Q}}^{\text {Yukawa }},
$$

as is indicated in the dual superconductor picture for color confinement. ${ }^{3,15}$ However, we obtain $m \simeq 0$ for the screening mass $m$, which would support 
the type-II dual superconductor, ${ }^{15}$ if this picture is correct. In any case, the Coulomb plus Y-type linear potential is confirmed once again. ${ }^{7}$

\subsection{Other Recent Studies on the $3 Q$ Potential}

To clarify the current status of the $3 \mathrm{Q}$ potential, we introduce two recent studies on the $3 \mathrm{Q}$ potential.

de Forcrand's group : Recently, de Forcrand's group, who supported $\Delta$-Ansatz in lattice QCD, ${ }^{16}$ seems to change their opinion from $\Delta$-Ansatz to Y-Ansatz ${ }^{17}$ except for a very short distance, where the linear potential seems negligible compared with the Coulomb contribution. (As a problem of their argument, they relied on the continuum Coulomb potential even for the subtle argument at the very short distance, where the lattice Coulomb potential should be used.)

Cornwall : One of the theoretical basis of $\Delta$-Ansatz was Cornwall's conjecture based on the vortex vacuum model. ${ }^{18}$ Very recently, motivated by our studies, Cornwall re-examined his previous work and found an error in his model calculation. His corrected answer is Y-Ansatz instead of $\Delta$-Ansatz. ${ }^{19}$

In this way, Y-Ansatz for the static $3 \mathrm{Q}$ potential seems almost settled both in lattice QCD and in analytic framework.

\section{Y-type Flux-Tube Formation in Lattice QCD}

Recently, as a clear evidence for Y-Ansatz, Y-type flux-tube formation is actually observed in the maximally-Abelian projected QCD from the direct measurement for the action density of the gluon field in the spatially-fixed 3Q system. ${ }^{20,21,22}$ (See Fig.5)
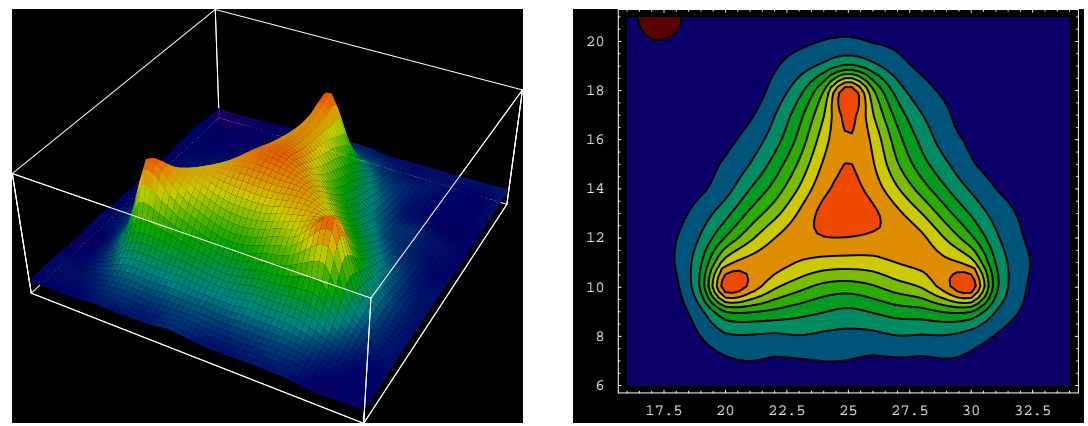

Figure 5. The lattice QCD result for Y-type flux-tube formation in the spatially-fixed 3Q system in maximally-Abelian projected QCD. The distance between the junction and each quark is about $0.5 \mathrm{fm}$. 


\section{The Excited-State Three-Quark Potential in QCD}

In 1969, Y. Nambu first pointed out the string picture for hadrons ${ }^{23,24}$ to explain the Veneziano amplitude ${ }^{25}$ on hadron reactions and resonances. Since then, the string picture has been one of the most important pictures for hadrons and has provided many interesting ideas in the wide region of the elementary particle physics.

For instance, the hadronic string creates infinite number of hadron resonances as the vibrational modes, and these excitations lead to the Hagedorn "ultimate" temperature ${ }^{26}$ which gives an interesting theoretical picture for the QCD phase transition.

For the real hadrons, of course, the hadronic string is to have a spatial extension like the flux-tube, as the result of one-dimensional squeezing of the color-electric flux in accordance with color confinement. ${ }^{27}$ Therefore, the vibrational modes of the hadronic flux-tube should be much more complicated, and the analysis of the excitation modes is important to clarify the underlying picture for real hadrons.

In the language of $\mathrm{QCD}$, such non-quark-origin excitation is called as the "gluonic excitation", and is physically interpreted as the excitation of the gluon-field configuration in the presence of the quark-antiquark pair or the three quarks in a color-singlet state.

In the hadron physics, the gluonic excitation is one of the interesting phenomena beyond the quark model, and relates to the hybrid hadrons such as $q \bar{q} G$ and $q q q G$. In particular, the hybrid meson includes the exotic hadrons with $J^{P C}=0^{--}, 0^{+-}, 1^{-+}, 2^{+-}, \cdots$, which cannot be constructed within the simple quark model.

In this section, we study the excited-state $3 \mathrm{Q}$ potential and the gluonic excitation using lattice $\mathrm{QCD},{ }^{8}$ to get deeper insight on these excitations beyond the hypothetical models such as the string and the flux-tube models. Here, the excited-state 3Q potential is the energy of the excited state of the gluon-field configuration in the presence of the static three quarks, and the gluonic-excitation energy is expressed as the energy difference between the ground-state $3 \mathrm{Q}$ potential and the excited-state $3 \mathrm{Q}$ potential.

\subsection{Formalism to extract Excited-State $3 Q$ Potentials}

We present the formalism to extract the excited-state potential. ${ }^{8}$ For the simple notation, the ground state is regarded as the "0-th excited state". For the physical eigenstates of the QCD Hamiltonian $\hat{H}$ for the spatiallyfixed 3Q system, we denote the $n$-th excited state by $|n\rangle(n=0,1,2, \cdots)$. Since the three quarks are spatially fixed in this case, the eigenvalue of $\hat{H}$ is 
expressed by a static potential as $\hat{H}|n\rangle=V_{n}|n\rangle$, where $V_{n}$ denotes the $n$-th excited-state $3 \mathrm{Q}$ potential. Note that both $V_{n}$ and $|n\rangle$ are universal physical quantities relating to the QCD Hamiltonian $\hat{H}$. In fact, $V_{n}$ depends only on the 3Q location, and $|n\rangle$ satisfies the orthogonal condition as $\langle m \mid n\rangle=\delta_{m n}$.

Suppose that $\left|\Phi_{k}\right\rangle(k=0,1,2, \cdots)$ are arbitrary given independent spatially-fixed 3Q states. In general, each 3Q state $\left|\Phi_{k}\right\rangle$ can be expressed with a linear combination of the $3 \mathrm{Q}$ physical eigenstates $|n\rangle$ as

$$
\left|\Phi_{k}\right\rangle=c_{0}^{k}|0\rangle+c_{1}^{k}|1\rangle+c_{2}^{k}|2\rangle+\cdots .
$$

Here, the coefficients $c_{n}^{k}$ depend on the selection of $\left|\Phi_{k}\right\rangle$, and hence they are not universal quantities.

The Euclidean-time evolution of the 3Q state $\left|\Phi_{k}(t)\right\rangle$ is expressed with the operator $e^{-\hat{H} t}$, which corresponds to the transfer matrix in lattice QCD. The overlap $\left\langle\Phi_{j}(T) \mid \Phi_{k}(0)\right\rangle$ is given by the 3Q Wilson loop with the initial state $\left|\Phi_{k}\right\rangle$ at $t=0$ and the final state $\left|\Phi_{j}\right\rangle$ at $t=T$, and is expressed in the Euclidean Heisenberg picture as

$$
\begin{aligned}
W_{T}^{j k} & \equiv\left\langle\Phi_{j}\left|W_{3 Q}(T)\right| \Phi_{k}\right\rangle=\left\langle\Phi_{j}(T) \mid \Phi_{k}(0)\right\rangle=\left\langle\Phi_{j}\left|e^{-\hat{H} T}\right| \Phi_{k}\right\rangle \\
& =\sum_{m=0}^{\infty} \sum_{n=0}^{\infty} \bar{c}_{m}^{j} c_{n}^{k}\left\langle m\left|e^{-\hat{H} T}\right| n\right\rangle=\sum_{n=0}^{\infty} \bar{c}_{n}^{j} c_{n}^{k} e^{-V_{n} T} .
\end{aligned}
$$

Using the matrix $C$ satisfying $C^{n k}=c_{n}^{k}$ and the diagonal matrix $\Lambda_{T}$ as $\Lambda_{T}^{m n}=e^{-V_{n} T} \delta^{m n}$, we rewrite the above relation as

$$
W_{T}=C^{\dagger} \Lambda_{T} C \text {. }
$$

Note here that $C$ is not a unitary matrix, and hence this relation does not mean the simple diagonalization by the unitary transformation.

Since we are interested in the $3 \mathrm{Q}$ potential $V_{n}$ in $\Lambda_{T}$ rather than the non-universal matrix $C$, we single out $V_{n}$ from the $3 \mathrm{Q}$ Wilson loop $W_{T}$ as

$$
W_{T}^{-1} W_{T+1}=\left\{C^{\dagger} \Lambda_{T} C\right\}^{-1} C^{\dagger} \Lambda_{T+1} C=C^{-1} \operatorname{diag}\left(e^{-V_{0}}, e^{-V_{1}}, \cdots\right) C,
$$

which is a similarity transformation. Then, $e^{-V_{n}}$ can be obtained as the eigenvalues of the matrix $W_{T}^{-1} W_{T+1}$, i.e., solutions of the secular equation,

$$
\operatorname{det}\left\{W_{T}^{-1} W_{T+1}-t \mathbf{1}\right\}=\prod_{n}\left(e^{-V_{n}}-t\right)=0 .
$$

Thus, the $3 \mathrm{Q}$ potential $V_{n}$ can be obtained from the matrix $W_{T}^{-1} W_{T+1}$.

In the practical calculation, we prepare $N$ independent sample states $\left|\Phi_{k}\right\rangle(k=0,1, \cdots, N-1)$. By choosing appropriate states $\left|\Phi_{k}\right\rangle$ so as not to include highly excited-state components, the physical states $|n\rangle$ can be 
truncated as $0 \leq n \leq N-1$. Then, $W_{T}, C$ and $\Lambda_{T}$ are reduced into $N \times N$ matrices, and the secular equation (13) becomes the $N$-th order equation.

\subsection{Lattice $Q C D$ results for the Excited-State $3 Q$ Potential}

For more than 100 different patterns of spatially-fixed 3Q systems, we study the excited-state potential $V_{3 \mathrm{Q}}^{\text {e.s. }}$ using lattice $\mathrm{QCD}$ with $16^{3} \times 32$ at $\beta=5.8$ and 6.0 at the quenched level. ${ }^{8}$ In Fig.6, we show the first lattice QCD results for the excited-state $3 \mathrm{Q}$ potential $V_{3 \mathrm{Q}}^{\text {e.s. }}$ as well as the ground-state potential $V_{3 \mathrm{Q}}^{\text {g.s. }}$. (In Fig.6, the minimal length $L_{\min }$ of the Y-type flux-tube is used as a label to distinguish the three-quark configuration.)

The energy gap between $V_{3 \mathrm{Q}}^{\text {g.s. }}$ and $V_{3 \mathrm{Q}}^{\text {e.s. }}$ physically means the excitation energy of the gluon-field configuration in the presence of the spatially-fixed three quarks, and the gluonic excitation energy $\Delta E_{3 \mathrm{Q}} \equiv V_{3 \mathrm{Q}}^{\text {e.s. }}-V_{3 \mathrm{Q}}^{\text {g.s. }}$ is found to be about $1 \mathrm{GeV}$ or more ${ }^{8,21,22}$ in the typical hadronic scale as $L_{\min } \sim 1 \mathrm{fm}$.

Note that the gluonic excitation energy of about $1 \mathrm{GeV}$ is rather large in comparison with the excitation energies of the quark origin, and such a gluonic excitation would contribute significantly in the highly-excited baryons with the excitation energy above $1 \mathrm{GeV}$. The present result predicts that the lowest hybrid baryon, which is described as $q q q G$ in the valence picture, has a large mass of about $2 \mathrm{GeV} .^{8}$

Together with the recent lattice result ${ }^{10}$ indicating the gluonic excitation energy $\Delta E_{\mathrm{Q} \overline{\mathrm{Q}}} \equiv V_{\mathrm{Q} \overline{\mathrm{Q}}}^{\text {e.s. }}-V_{\mathrm{Q} \overline{\mathrm{Q}}}^{\mathrm{g} \text {.s. }}$ for the $\mathrm{Q}-\overline{\mathrm{Q}}$ system to be in the order of $1 \mathrm{GeV}$, the present result seems to suggest the constituent gluon mass of about $1 \mathrm{GeV}$ in terms of the constituent gluon picture.
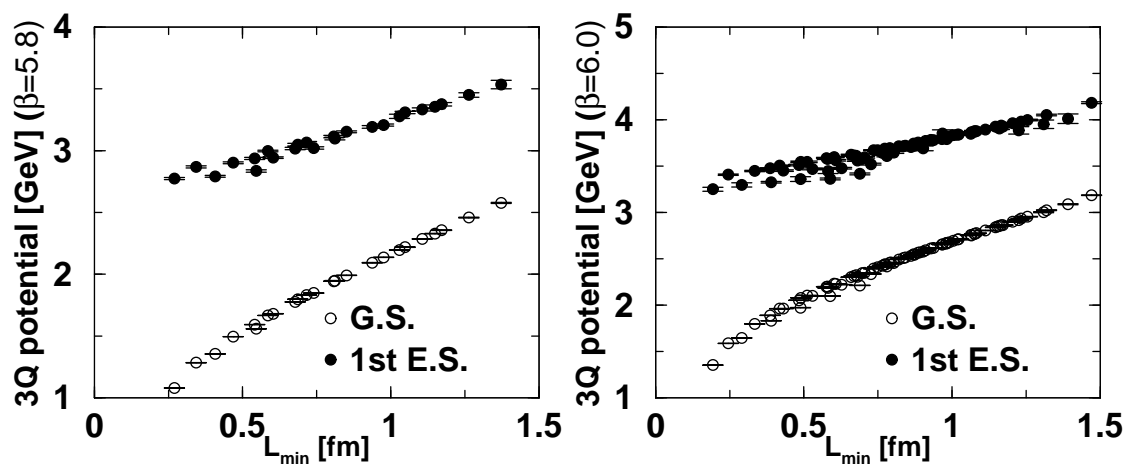

Figure 6. The lattice QCD results of the ground-state $3 \mathrm{Q}$ potential $V_{3 \mathrm{Q}}^{\text {g.s. }}$ (open circles) and the 1 st excited-state $3 \mathrm{Q}$ potential $V_{3 \mathrm{Q}}^{\text {e.s. }}$ (filled circles) as the function of $L_{\mathrm{min}}$. These lattice results at $\beta=5.8$ and $\beta=6.0$ well coincide besides an irrelevant overall constant. The gluonic excitation energy $\Delta E_{3 \mathrm{Q}} \equiv V_{3 \mathrm{Q}}^{\text {e.s. }}-V_{3 \mathrm{Q}}^{\text {g.s. }}$ is found to be about $1 \mathrm{GeV}$ in the hadronic scale as $L_{\text {min }} \sim 1 \mathrm{fm}$. 


\subsection{Functional Form of the Excited-State $3 Q$ Potential and Comparison with the $Q-\bar{Q}$ System}

From the lattice QCD data, we attempt to seek the functional form of the excited-state $3 \mathrm{Q}$ potential $V_{3 \mathrm{Q}}^{\text {e.s. }}$, but find no simple plausible form of $V_{3 \mathrm{Q}}^{\text {e.s. }}$, unlike the ground-state $3 \mathrm{Q}$ potential $V_{3 \mathrm{Q}}^{\text {g.s. }}$.

Next, we compare the $3 \mathrm{Q}$ gluonic excitation with the Q- $\bar{Q}$ gluonic excitation, considering the nature of the Y-type junction. If the Y-type junction behaves as a quasi-fixed edge of the three flux-tubes, these three flux-tubes would behave as independent three Q- $\overline{\mathrm{Q}}$ systems, and therefore the $3 \mathrm{Q}$ gluonic excitation would be approximated as a simple incoherent sum of the three $\mathrm{Q}-\overline{\mathrm{Q}}$ gluonic excitations. If the Y-type junction behaves as a quasifree edge, the $3 \mathrm{Q}$ gluonic-excitation energy $\Delta E_{3 \mathrm{Q}}$ would be smaller than each of $\mathrm{Q}-\overline{\mathrm{Q}}$ gluonic-excitation energies $\Delta E_{\mathrm{Q}} \overline{\mathrm{Q}}$ corresponding to the three flux-tubes, since the string with fixed edges has a larger vibrational energy.

Through the comparison of $V_{3 \mathrm{Q}}^{\text {e.s. }}$ or $\Delta E_{3 \mathrm{Q}}$ with several possible linear combinations of $V_{\mathrm{Q} \overline{\mathrm{Q}}}^{\text {e.S. }}$ or $\Delta E_{\mathrm{Q} \overline{\mathrm{Q}}}$, we find no simple relation between them. This fact is conjectured to reflect the complicated vibrational mode on the Y-type flux-tube, due to the interference among the vibrational modes on the three flux-tubes through the junction, which may indicate the quasi-free behavior of the Y-type junction.

\section{Behind the Success of the Quark Model}

Finally, we consider the connection between QCD and the quark model in terms of the gluonic excitation. ${ }^{8,21,22}$ While QCD is described with quarks and gluons, the simple quark model successfully describes low-lying hadrons even without explicit gluonic modes. In fact, the gluonic excitation seems invisible in the low-lying hadron spectra, which is rather mysterious.

On this point, we find the gluonic-excitation energy to be about $1 \mathrm{GeV}$ or more, which is rather large compared with the excitation energies of the quark origin, and therefore the effect of gluonic excitations is negligible and quark degrees of freedom plays the dominant role in low-lying hadrons with the excitation energy below $1 \mathrm{GeV}$.

Thus, the large gluonic-excitation energy of about $1 \mathrm{GeV}$ gives the physical reason for the invisible gluonic excitation in low-lying hadrons, which would play the key role for the success of the quark model without gluonicexcitation modes. ${ }^{8,21,22}$

In Fig.7, by way of the flux-tube picture, we present a possible scenario from QCD to the massive quark model in terms of color confinement and dynamical chiral-symmetry breaking. ${ }^{21,22}$ 


\section{Summary and Concluding Remarks}

Using SU(3) lattice QCD, we have studied the ground-state 3Q potential $V_{3 \mathrm{Q}}^{\text {g.s. }}$ and the 1 st excited-state $3 \mathrm{Q}$ potential $V_{3 \mathrm{Q}}^{\text {e.s. }}$.

From the accurate and thorough calculation for more than 300 different patterns of $3 \mathrm{Q}$ systems, we have found that the static ground-state $3 \mathrm{Q}$ potential $V_{3 \mathrm{Q}}^{\text {g.s. }}$ is well described by the Coulomb plus Y-type linear potential, i.e., Y-Ansatz, within 1\%-level deviation. As a clear evidence for Y-Ansatz, Y-type flux-tube formation has been actually observed on the lattice in maximally-Abelian projected QCD.

For more than 100 different patterns of 3Q systems, we have performed the first study of the 1 st excited-state $3 \mathrm{Q}$ potential $V_{3 \mathrm{Q}}^{\text {e.s. }}$ in quenched lattice

\section{Quantum Chromodynamics}
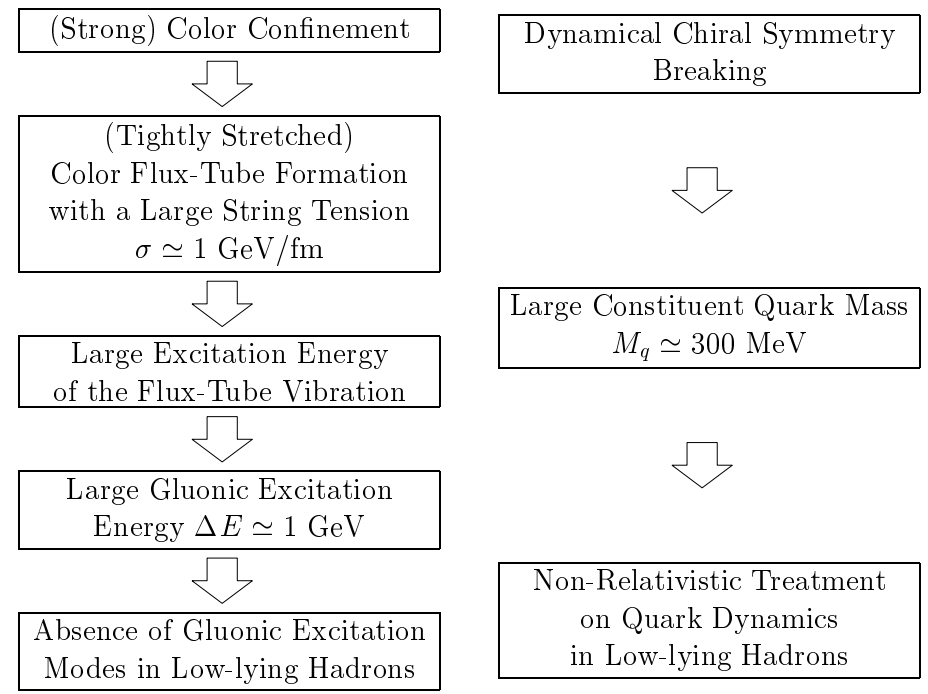

\section{Massive Quark Model for Low-lying Hadrons}

Figure 7. A possible scenario from QCD to the quark model in terms of color confinement and dynamical chiral-symmetry breaking (DCSB). DCSB provides a large constituent quark mass of about $300 \mathrm{MeV}$, which enables the non-relativistic treatment for quark dynamics. Color confinement provides the color flux-tube formation among quarks with a large string tension of $\sigma \simeq 1 \mathrm{GeV} / \mathrm{fm}$. In the flux-tube picture, the gluonic excitation is described as the flux-tube vibration, and the flux-tube vibrational energy is expected to be large, reflecting the large string tension. The large gluonic-excitation energy of about $1 \mathrm{GeV}$ leads to the absence of the gluonic mode in low-lying hadrons, which would play the key role to the success of the quark model without gluonic excitation modes. 
$\mathrm{QCD}$, and have found the gluonic excitation energy $\Delta E_{3 \mathrm{Q}} \equiv V_{3 \mathrm{Q}}^{\text {e.s. }}-V_{3 \mathrm{Q}}^{\text {g.s. }}$ to be about $1 \mathrm{GeV}$. This indicates that the hybrid baryons $(q q q G)$ are to be rather heavy and appear in the spectrum above $2 \mathrm{GeV}$. We have conjectured that the large gluonic-excitation energy of about $1 \mathrm{GeV}$ leads to the success of the quark model for the low-lying hadrons even without gluonic excitations.

Acknowledgements H.S. thanks all the participants of Confinement2003.

\section{References}

1. Y. Nambu, in Preludes in Theoretical Physics, in honor of V.F. Weisskopf (North-Holland, Amsteldam,1966).

2. M.Y. Han and Y. Nambu, Phys. Rev. 139, B1006 (1965).

3. For instance, articles in Quantum Chromodynamics and Color Confinement, edited by H. Suganuma, M. Fukushima and H. Toki (World Scientific, 2001).

4. Y. Nambu, G. Juna-Lasinio, Phys. Rev. 122, 345 (1961); ibid. 124, 246 (1961).

5. H.J. Rothe, Lattice Gauge Theories, 2nd edition (World Scientific, 1997) p.1.

6. T.T. Takahashi, H. Matsufuru, Y. Nemoto and H. Suganuma, Phys. Rev. Lett. 86, 18 (2001).

7. T.T. Takahashi, H. Suganuma, Y. Nemoto and H. Matsufuru, Phys. Rev. D65, 114509 (2002) and references therein.

8. T.T. Takahashi and H. Suganuma, Phys. Rev. Lett. 90, 182001 (2003).

9. T.T. Takahashi, H. Matsufuru, Y. Nemoto and H. Suganuma, Proc. of the TMU-Yale Symp. on Dynamics of Gauge Fields, Tokyo, Dec. 1999, edited by A. Chodos et al., (Universal Academy Press, 2000) 179.

10. K.J. Juge, J. Kuti and C.J. Morningstar, Phys. Rev. Lett. 90, 161601 (2003).

11. J. Kogut and L. Susskind, Phys. Rev. D11, 395 (1975).

12. S. Capstick and N. Isgur, Phys. Rev. D34, 2809, (1986).

13. M. Fable de la Ripelle and Yu. A. Simonov, Ann. Phys. 212, 235 (1991).

14. N. Brambilla, G.M. Prosperi and A. Vairo, Phys. Lett. B362, 113 (1995).

15. H. Suganuma, S. Sasaki and H. Toki, Nucl. Phys. B435, 207 (1995).

16. C. Alexandrou, P. de Forcrand, A. Tsapalis, Phys. Rev. D65, 054503 (2002).

17. O. Jahn and P. de Forcrand, Proc. of Lattice 2003, hep-lat/0309115.

18. J.M. Cornwall, Phys. Rev. D54, 6527 (1996).

19. J.M. Cornwall, "On the Center-Vortex Baryonic Area Law", hep-th/0305101.

20. H. Ichie, V. Bornyakov, T. Streuer and G. Schierholz, Nucl. Phys. A721, 899 (2003); Nucl. Phys. B (Proc.Suppl.) 119, 751 (2003).

21. T.T. Takahashi, H. Suganuma, H. Ichie, H. Matsufuru and Y. Nemoto, Nucl. Phys. A721, 926 (2003).

22. H. Suganuma, T.T. Takahashi and H. Ichie, Nucl. Phys. A in press.

23. Y. Nambu, in Symmetries and Quark Models (Wayne State University, 1969).

24. Y. Nambu, Lecture Notes at the Copenhagen Symposium (1970).

25. G. Veneziano, Nuovo Cim. A57, 190 (1968).

26. R. Hagedorn, Nuovo Cim. Suppl. 3, 147 (1965).

27. Y. Nambu, Phys. Rev. D10, 4262 (1974). 